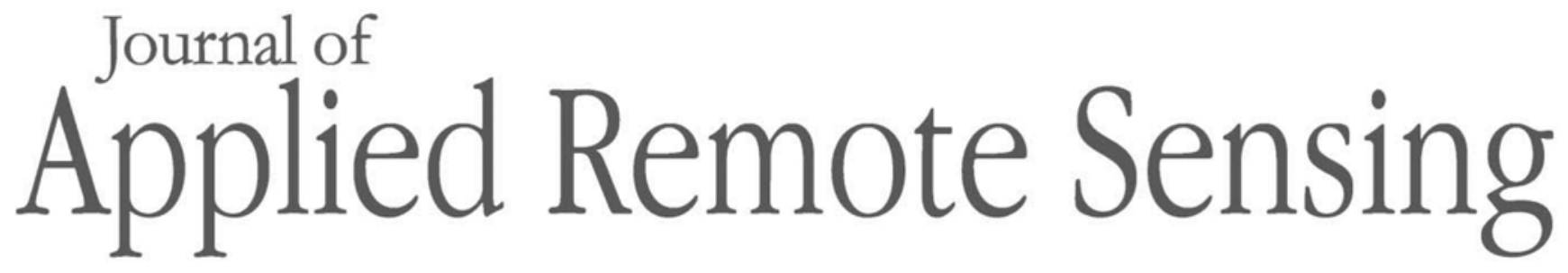

RemoteSensing.SPIEDigitalLibrary.org

\title{
Implications of spectral and spatial features to improve the identification of specific classes
}

\author{
Akhil Kallepalli \\ Anil Kumar \\ Kourosh Khoshelham \\ David B. James \\ Mark A. Richardson
}




\title{
Implications of spectral and spatial features to improve the identification of specific classes
}

\author{
Akhil Kallepalli, ${ }^{a} *$ Anil Kumar, ${ }^{\text {b }}$ Kourosh Khoshelham, ${ }^{c}$ \\ David B. James, ${ }^{a}$ and Mark A. Richardson ${ }^{a}$ \\ ${ }^{a}$ Defence Academy of the United Kingdom, Cranfield University, Centre for Electronic Warfare, \\ Information and Cyber, Shrivenham, United Kingdom \\ ${ }^{\mathrm{b}}$ ISRO, Govt. of India, Indian Institute of Remote Sensing, Photogrammetry and \\ Remote Sensing Department, Dehradun, India \\ ${ }^{c}$ University of Melbourne, Department of Infrastructure Engineering, Melbourne, Australia
}

\begin{abstract}
Dimensionality is one of the greatest challenges when deciphering hyperspectral imaging data. Although the multiband nature of the data is beneficial, algorithms are faced with a high computational load and statistical incompatibility due to the insufficient number of training samples. This is a hurdle to downstream applications. The combination of dimensionality and the real-world scenario of mixed pixels makes the identification and classification of imaging data challenging. Here, we address the complications of dimensionality using specific spectral indices from band combinations and spatial indices from texture measures for classification to better identify the classes. We classified spectral and combined spatialspectral data and calculated measures of accuracy and entropy. A reduction in entropy and an overall accuracy of $80.50 \%$ was achieved when using the spectral-spatial input, compared with $65 \%$ for the spectral indices alone and $59.50 \%$ for the optimally determined principal components. (C) 2019 Society of Photo-Optical Instrumentation Engineers (SPIE) [DOI: 10.1117/1 .JRS.13.016504]
\end{abstract}

Keywords: hyperspectral imaging; vegetation analysis; classification algorithms; mixed pixels; urban scene classification.

Paper 180601 received Jul. 16, 2018; accepted for publication Dec. 14, 2018; published online Jan. 14, 2019.

\section{Introduction}

A target's spectral information can be acquired in many, contiguous bands of specific wavelengths, a phenomenon known as hyperspectral imaging ${ }^{1,2}$ Due to the nature of the information acquired (specific, narrow bandwidths detecting minor changes of reflectance), the datasets usually have intrinsic diagnostic characteristics with respect to the target. ${ }^{3,4}$ The identification of targets can be achieved using variations in spectral responses that can be attributed to situational, conditional, or illumination changes.

Most hyperspectral applications and assessments begin by addressing the dimensionality of the dataset. Spectral information is reduced using a preprocessing method to either identify features on the basis of interest or "project" them into a feature space based on the amount of valuable information. Even when attributing credibility to the narrow-bandwidth, contiguous bands for greater spectral information, the datasets also encounter the "curse of dimensionality." Usually referred to as the Hughes Phenomenon, ${ }^{5}$ the statistical accuracy of class recognition is known to optimize for a subset of bands and subsequently decline due to inadequate training samples. ${ }^{6}$ The benefits of dimensionality reduction including the lower computational load are widely accepted. ${ }^{1,3,7}$

Extending the current literature, we gain insight into the implications of using spectral and spatial information as the input database for the classification algorithm instead of an output of feature extraction. This paper reports the results of knowledge-based feature selection and

*Address all correspondence to Akhil Kallepalli, E-mail: a.kallepalli@cranfield.ac.uk

$1931-3195 / 2019 / \$ 25.00$ (C) 2019 SPIE 
transformation to indices for the improvement of identification for specific classes. A possibilistic $c$-means (PCM) classifier was used due to its advantages over other mixed pixel classifiers, i.e., the probability of classes within a pixel are not constrained. ${ }^{8}$ We applied the classifier to the Airborne Prism Experiment (APEX) Open Science Dataset (OSD). To demonstrate the improvement in the identification accuracy of individual classes, a baseline classification was established using principal components analysis (PCA) dimensionality reduction to establish entropy for the statistical quantification of uncertainty. ${ }^{6}$ The classification approach is based on our earlier report $^{9}$ and targeted the identification of specific, individual classes and the improvements were used to validate the results.

\section{Related Work}

Light is reflected in characteristic ways from targets that allow their differentiation. Hyperspectral data, exploiting this characteristic variation, has found many applications including its use to determine the extent of vegetation for health analysis, ${ }^{10}$ species-level classification, ${ }^{11}$ and biomedical research. ${ }^{12}$ However, the additional spectral information reduces the classification accuracy and results in a high computational load. This phenomenon has been described as a statistical inadequacy of training samples compared with the number of bands in the dataset. ${ }^{5}$ A "peaking phenomenon" was said to occur, with accuracy improving for a subset of bands and thereafter declining with every additional band. Although this phenomenon has been challenged as apparent in nature, ${ }^{13}$ the reduction of hyperspectral data dimensionality is nevertheless a key step in the application of imaging data. To avoid the problems associated with dimensionality, numerous methods have been applied to reduce the dataset prior to information extraction.

Information from higher dimensional imaging data requires methods that can broadly be categorized as feature selection and feature extraction. Feature selection methods refer to algorithms that output a subset of the original dimensionality, whereas feature extraction methods create new features either by transformation or by combining input features.$^{14}$ Methods such as $\mathrm{PCA}^{15,16}$ use linear transformation to convert the data into a reduced or "intrinsic dimensionality." ${ }^{17}$ Such transformations are not universally applicable. An attempt has been made to optimize the accuracy of the feature selection method using sequential forward selection, steepest ascent, and fast constrained search strategies. ${ }^{18}$ Another approach attempted simultaneously to reduce the dimensionality and classify the dataset by projecting the signals into orthogonal subspace, eliminating unwanted signatures, and improving signal-to-noise ratios. ${ }^{7}$ In another, a sequence of morphological transformations was used to filter and classify highly dimensional data. ${ }^{19}$ As well as reducing the computational load, dimensionality reduction greatly improves the classification accuracy. It results in a comparable number of training samples against a subset of bands with the information from the original image. However, PCA and similar methods have several disadvantages: they assume the data dimensionality can be reduced by linear transformation when data could be nonlinear in relation, and the initial components are dominated by data showing greater variance while not (with exceptions) maximizing the extracted information. ${ }^{15}$ These disadvantages have prompted further research into nonlinear and/or knowledge-based dimensionality reduction.

\subsection{Pixel-Based Spectral Indices}

Emphasizing the importance of obtaining useful information from the image data, methods of feature selection can be employed or combinations of bands (feature extraction) can be used. Spectral indices are derived from distinctive characteristics in the spectral signatures of the target to highlight them against the background. For example, biophysical variables can be quantified by remote sensing ${ }^{20}$ with the identification of color signatures in individual spectral bands and grayscale texture analysis. ${ }^{21-23}$ The formulation of spectral indices began with the simple ratio (SR $)^{24}$ of bands. The contrasting spectral response (Fig. 1) in the infrared $(0.8 \mu \mathrm{m})$ and red $(0.675 \mu \mathrm{m})$ wavelength regions was used by defining the ratio of individual pixel values. 


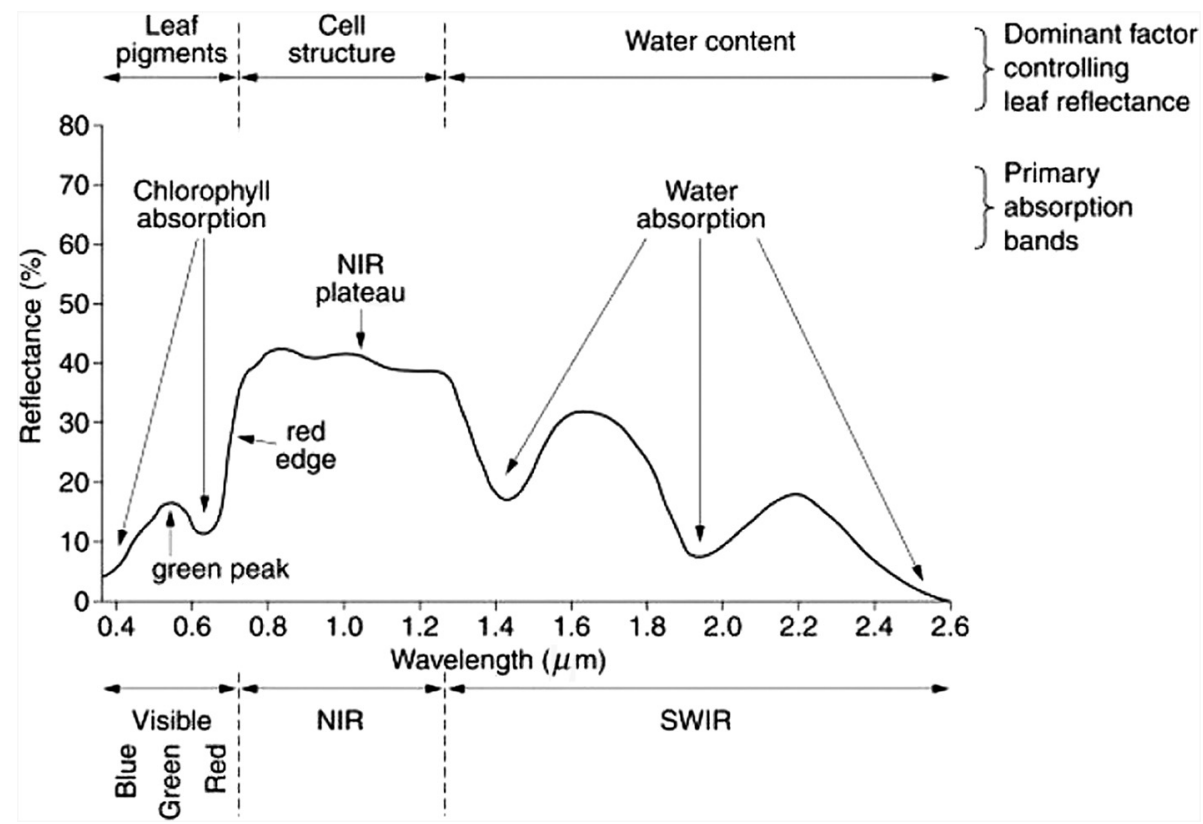

Fig. 1 Identifiable biophysical aspects correlated with spectral information. ${ }^{25}$

One of the most popular spectral indices is the normalized difference vegetation index (NDVI) ${ }^{26}$ which is a modified version of the SR index. The ratio of the reflectance values $(R)$ in the near-infrared and red bands $\left[\left(R_{\mathrm{NIR}}-R_{R}\right) /\left(R_{\mathrm{NIR}}+R_{R}\right)\right.$ for Landsat 1 data] was applied in NDVI to enhance vegetation features compared to the background, producing results in the grayscale values. ${ }^{27}$ Although the delineation of vegetation features by NDVI was satisfactory, the correlation between different vegetation parameters and the underlying soil reflectance affects the results. ${ }^{28}$ To address these vegetation-specific aspects, many indices have been formulated to account for the chlorophyll content, ${ }^{29-31}$ leaf area index (LAI), ${ }^{32-34}$ and other parameters.

Interference caused by the soil was addressed by the development of the soil adjusted vegetation index (SAVI), which is shown in Eq. (1): $:^{28}$

$$
\mathrm{SAVI}=\frac{\left(R_{\mathrm{NIR}}-R_{R}\right)}{\left(R_{\mathrm{NIR}}+R_{R}+L\right)} \times(1+L)
$$

where $L$ defines the prior knowledge of the vegetation density, i.e., low vegetation $(L=1)$, intermediate vegetation cover $(L=0.5)$, and high-density vegetation cover $(L=0.25)$. The need for prior knowledge of vegetation density cover was eliminated in the modified SAVI index (MSAVI), ${ }^{35}$ which is shown in Eq. (2):

$$
\text { MSAVI }=0.5\left[2 R_{\mathrm{NIR}}+1-\sqrt{\left(2 R_{\mathrm{NIR}}+1\right)^{2}-8\left(R_{\mathrm{NIR}}-R_{\mathrm{Red}}\right)}\right]
$$

The implications of narrowband data versus broadband data have been considered when estimating LAI and green cover. ${ }^{36}$ Applying the basic understanding of chlorophyll absorption in the red wavelength region and reflectance at NIR wavelengths, ${ }^{37}$ previously defined vegetation indices were explored to highlight the importance of narrow band hyperspectral imagery. LAI and canopy chlorophyll density were investigated using previously defined indices. $^{38}$

The chlorophyll absorption in reflectance index (CARI) ${ }^{39}$ was improved by developing a modified version (MCARI), ${ }^{34}$ which compares the reflectance at 0.67 to 0.55 and $0.7 \mu \mathrm{m}$ as shown in Eq. (3): 


$$
\operatorname{MCARI}=\left[\left(R_{0.7}-R_{0.67}\right)-0.2\left(R_{0.7}-R_{0.55}\right)\right] \times \frac{R_{0.7}}{R_{0.67}}
$$

The diagnostic capability of spectral indices and narrowband imaging was further highlighted through improved pigment and chlorophyll estimation ${ }^{40,41}$ in open-canopy tree crops, ${ }^{42}$ viticulture. ${ }^{43,44}$ This was done by measuring physiological characteristics using the physiological reflectance index, ${ }^{45}$ as well as the accumulation of specific metabolites such as carotenoids. ${ }^{46}$

The application of mathematical and/or ratio-based spectral indices to enhance certain features was also applied beyond the analysis of vegetation. The normalized difference built-up index (NDBI) ${ }^{47}$ was developed to classify built-up parts of urban areas utilizing the broadband data from the Landsat Thematic Mapper as shown in Eq. (4):

$$
\mathrm{NDBI}=\frac{\left(R_{\mathrm{TM} 5}-R_{\mathrm{TM} 4}\right)}{\left(R_{\mathrm{TM} 5}+R_{\mathrm{TM} 4}\right)},
$$

where TM5 is the shortwave infrared-1 band (1.55 to $1.75 \mu \mathrm{m})$ and TM4 is the NIR band (0.76 to $0.90 \mu \mathrm{m}$ ) of the Landsat Thematic Mapper.

Water bodies have been identified via their infrared absorption properties using the normalized difference water index (NDWI ${ }^{48}$ as shown in Eq. (5):

$$
\mathrm{NDWI}=\frac{\left(R_{0.86}-R_{1.24}\right)}{\left(R_{0.86}+R_{1.24}\right)}
$$

\subsection{Texture-Based Spatial Indices}

Although spatial indices can be interpreted in many ways, ${ }^{9}$ current research focuses on utilizing the spatial variation of pixels and the influence of a neighborhood of pixels on the detection of targets. Spatial textures are used to discriminate spectrally similar targets and are therefore applied to the spectral index output(s).

The primary pattern elements are spectral, textural, and contextual features. ${ }^{9}$ Whereas early research focused on the coarseness of features and edge definition, a discussion of textural features identified the important influence of pixel neighbourhoods. ${ }^{21}$ Derived textures used angular nearest neighborhood gray-tone spatial dependence matrices. ${ }^{21,49}$ The neighborhood of the pixels depend on the variation across pixels in the image. This correlates with the resolution of the image data and the extent of the targets. For example, if a large neighborhood is considered for a much smaller target (e.g., house roofs, as used in the current study), the target is lost in the

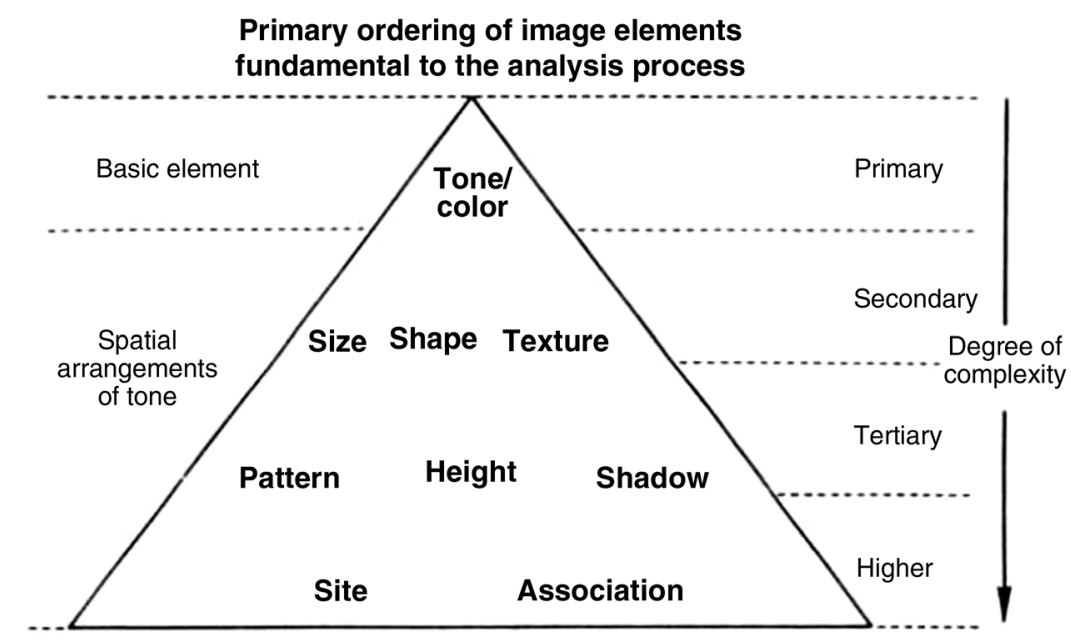

Fig. 2 Priority ordering of image elements, the basis of image analysis procedures. ${ }^{22}$ 
larger window of texture calculation. Alternatively, larger neighborhoods can be considered for targets (vegetation) that occupy a larger area of the image and show less spatial variation.

Texture is important for the accurate interpretation of images ${ }^{22}$ (Fig. 2). Applications have expanded to the interpretation of synthetic aperture radar imagery using gray-tone co-occurrence textural matrices. ${ }^{50}$ However, the most suitable texture analysis elements should be selected rather than using all elements, and this is the approach we have followed.

Texture analysis can provide quantitative discriminators along with spatial metrics ${ }^{49}$ to improve the identification of spatial information from datasets. We set out to improve the classification accuracy of datasets when using spatial and spectral information together as input to the classifier for identification of specific classes.

\section{Methods}

Many methods for the assessment of hyperspectral imaging for classification purposes perform dimensionality reduction before employing a classifier for target identification. ${ }^{1,3,7,16,19,51}$ The need for a versatile airborne imaging system resulted in the European Space Agency

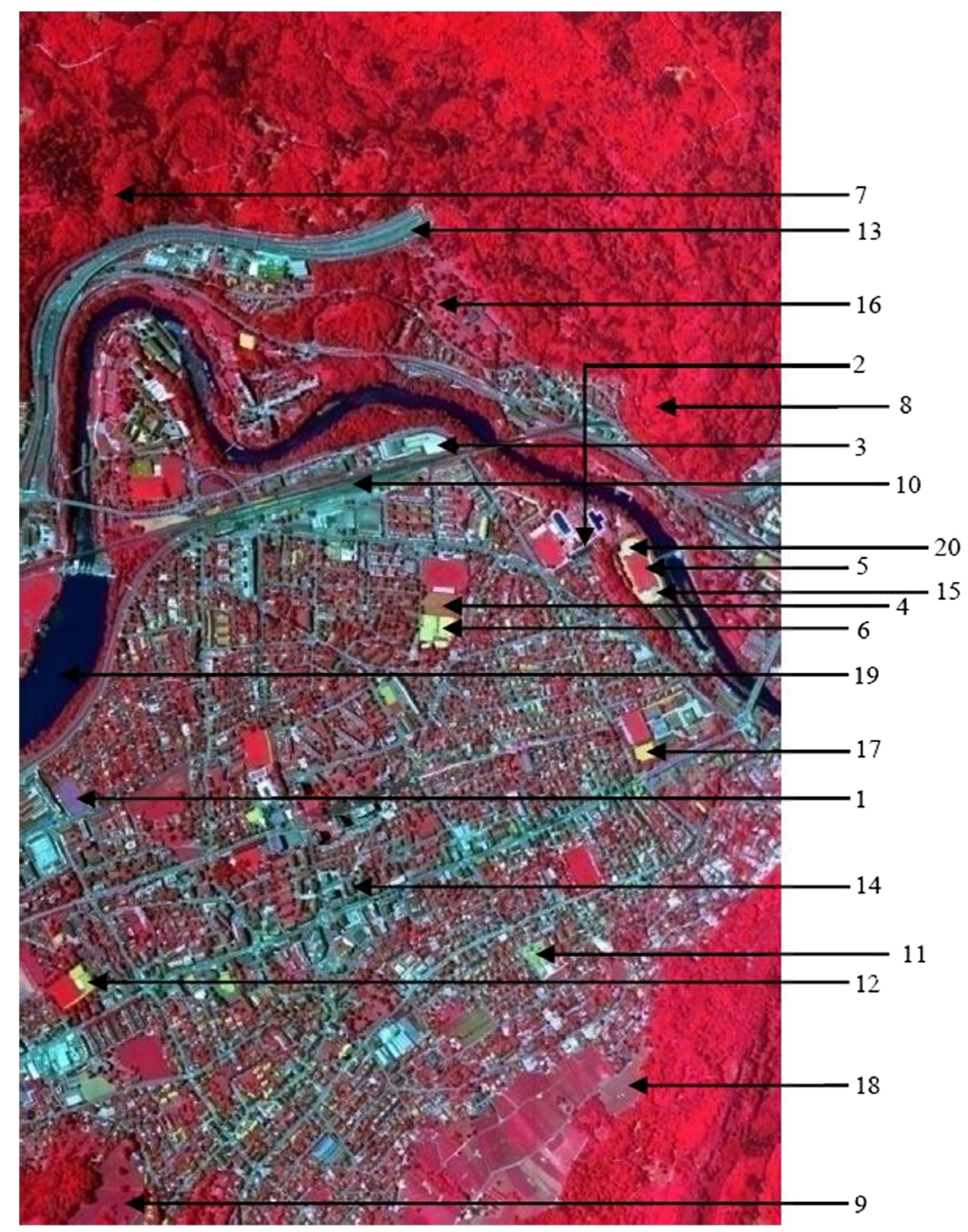

Fig. 3 APEX OSD false color composite image of Baden, Switzerland, ${ }^{56}$ showing representative areas of the different classes in the study. The classes are (1) artificial turf, (2) black roof, (3) building, (4) clay soil, (5) grass, (6) lawn tennis court, (7) coniferous forest, (8) deciduous forest, (9) pasture, (10) railway, (11) red roof, (12) red synthetic ground, (13) road, (14) roofs, (15) sand, (16) stressed grass, (17) synthetic sports surface, (18) vineyard, (19) water, and (20) yellow tartan. 
APEX project funded by Switzerland and Belgium. ${ }^{6,52}$ Detailed information about the APEX project can be found elsewhere. ${ }^{52-54}$

The APEX instrument operates in the range 0.38 to $2.5 \mu \mathrm{m}$ and can collect data in 313 freely configurable bands. ${ }^{55}$ The APEX OSD is freely available online at Ref. 56. Raw data acquired by the sensor in June 2011 were processed to a level 1 processed dataset. ${ }^{57}$ The spectral and spatial dimensions of the datasets are 285 bands and $1.8 \mathrm{~m}$, respectively. The spectral coverage of the dataset is in the range of 413 to $2421 \mathrm{~nm}$, across the 285 bands. The spectral sampling interval is 0.5 to $8 \mathrm{~nm}$ in the VNIR region and 10 to $6 \mathrm{~nm}$ in the SWIR. This results in a spectral resolution of 0.6 to $6.3 \mathrm{~nm}$ and 13.5 to $7 \mathrm{~nm}$, respectively, in the regions. The captured dataset included a variety of targets including grass, forests, roofs, and roads. The classes were identified using the SwissTopo web portal ${ }^{58}$ and specific pixels and regions of interest were shared by the data providers. The classes investigated were (1) artificial turf, (2) black roof, (3) building, (4) clay soil, (5) grass, (6) lawn tennis court, (7) coniferous forest, (8) deciduous forest, (9) pasture, (10) railway, (11) red roof, (12) red synthetic ground, (13) road, (14) roofs, (15) sand, (16) stressed grass, (17) synthetic sports surface, (18) vineyard, (19) water, and (20) yellow tartan. Training and testing datasets were developed and denoted in Fig. 3.

The "baseline" classification using PCA for dimensionality reduction was used to provide a means of comparison with the indices-based method. ${ }^{9}$ Entropy measures were used to ascertain the minimum number of principal components needed for classification. When more than seven components were present, there was no significant increase in classification accuracy to justify the increase of number of input bands to the PCM classifier. The classification and the accuracy assessment of the baseline classification (Fig. 4) were, therefore, achieved using seven components, establishing the overall baseline accuracy of $59.50 \%$.

The spectral signatures of each type of forest were similar, due to the presence of vegetation, but varied in terms of intensity [Fig. 5(a)]. This variation was caused by the difference of physical structure of coniferous and deciduous leaves, the latter having a larger surface area, and thus higher reflectance. Similar observations were recorded when comparing other classes [Fig. 5(b)].

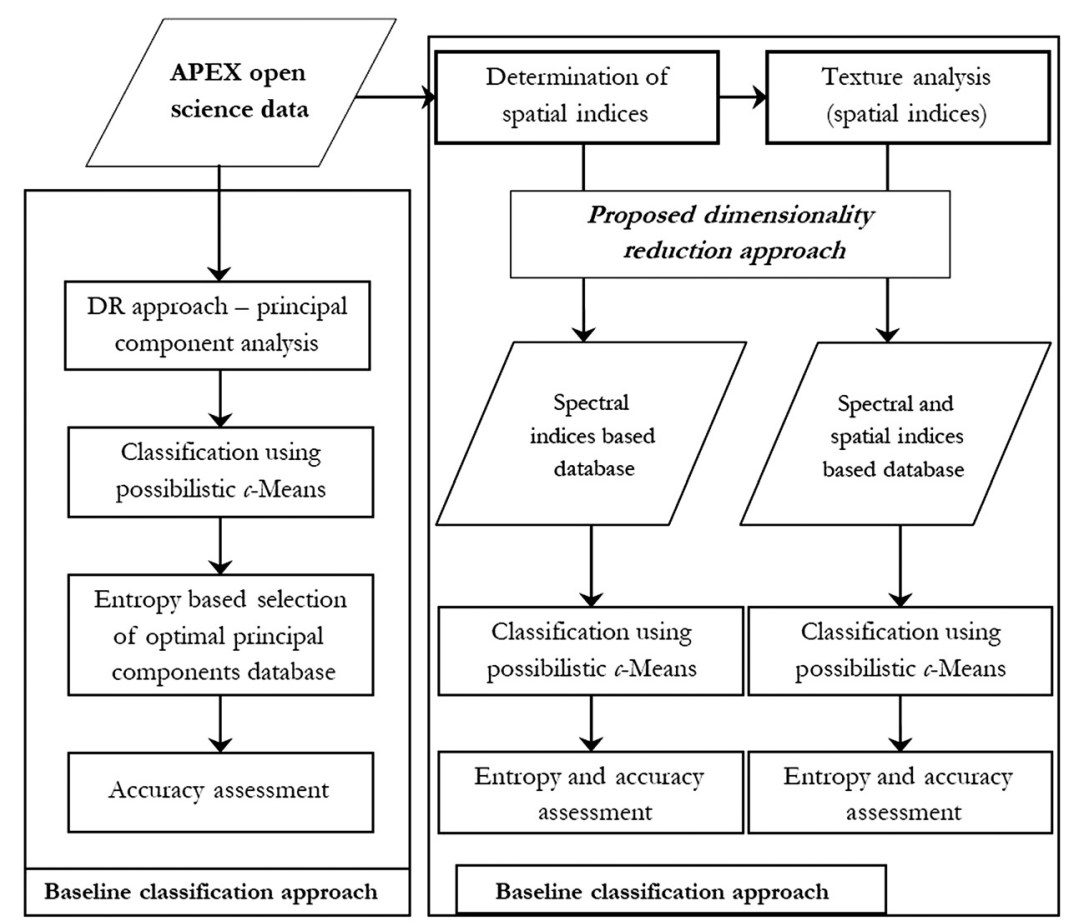

Fig. 4 The methodology followed in the study is presented. We would like to clarify that the proposed dimensionality reduction approach in this study is the knowledge-based identification of hyperspectral bands that will be used to evaluate the spectral indices. These identified spectral and spatial indices will be used as input to the classification algorithm. 


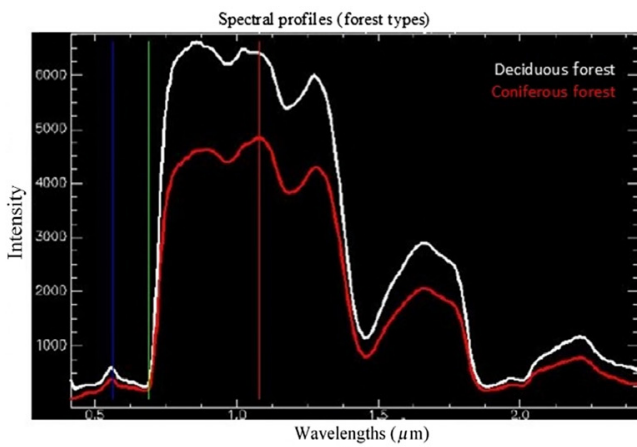

(a)

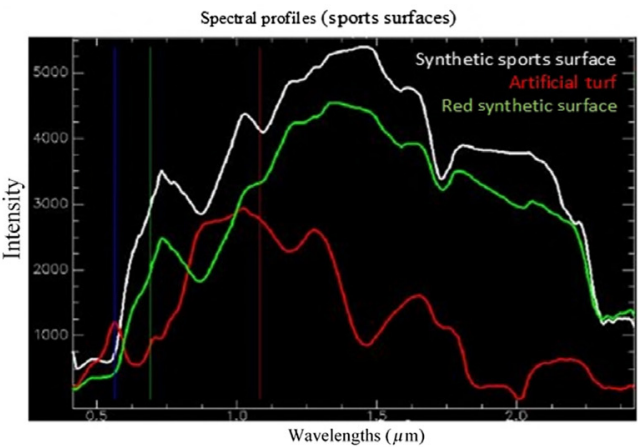

(c)

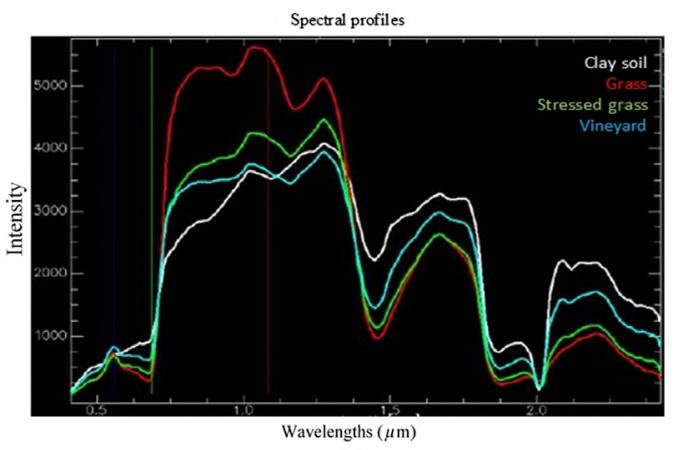

(b)

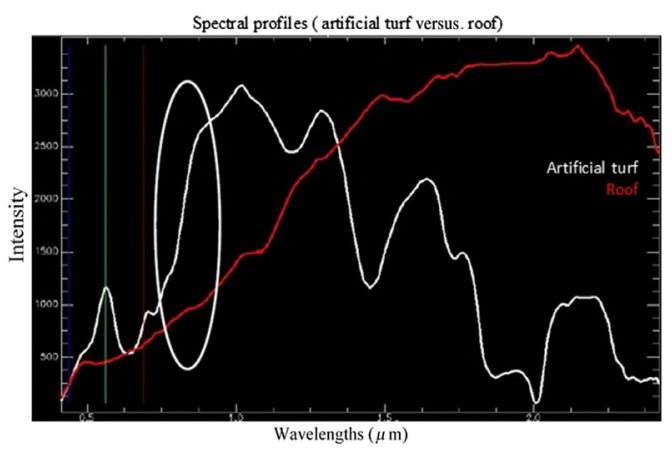

(d)

Fig. 5 Different spectral responses, plotted against intensity values, are shown in this image. The figures (a) Deciduous and coniferous forests; (b) soil, grass, stressed grass and vineyards; (c) synthetic sports surface, artificial turf and red synthetic ground; (d) artificial turfs and roofs. These spectral curves assist in identifying "keys" to differentiate the classes. These graphs were plotted using the ENVI image analysis software.

There were key differences in the spectral responses of healthy and stressed grass. The red edge represents absorption by leaf pigments and reflectance based on cell structure. The lack of leaf pigments in stressed grass contributes to the poorly developed red edge. When comparing the sports surfaces [Fig. 5(c)], the red sports surface shows distinguishable characteristics at $\approx 0.8 \mu \mathrm{m}$ when the spectral curve (shown in red) shows reflectance whereas the other two surfaces show absorption.

The reduction of reflectance and shift of red edge position (REP) toward the red wavelengths is indicative of less absorption by chlorophyll. The REP is estimated using the first derivative of the spectral response as a function of reflectance, yielding a specific band number of the red edge. ${ }^{59}$ The spectral curve of stressed grass illustrates a poorly defined red edge [Fig. 6(a)] with the first derivative of the spectral curve indicating a shift of REP [Fig. 6(b)] compared with the typical red edge for grass and the position of the red edge peak at the 64th band.

\subsection{Classification Approach}

When image data is collected from a target, the pixel is not composed of a single class in every situation. When more than one class is presented in a single pixel, it is considered to be "mixed." Classifiers that constrain a pixel to a single class are not suitable for the classification of mixed pixels. Soft or fuzzy classification approaches are better suited to such cases because they estimate the probability of a pixel belonging to a class, allowing multiple classes to have a degree of presence in a single pixel.

Fuzzy $c$-means (FCM) and possibilistic $c$-means (PCM) algorithms were considered for classification, but FCM was not suitable for this application because it only achieves dependable accuracy when all the classes are exhaustively defined (the membership function of the classifier is constrained to 1). In contrast, due to the lack of a membership function constraint, the PCM classifier better evaluates the presence of the class in a pixel as a function of its signature without 


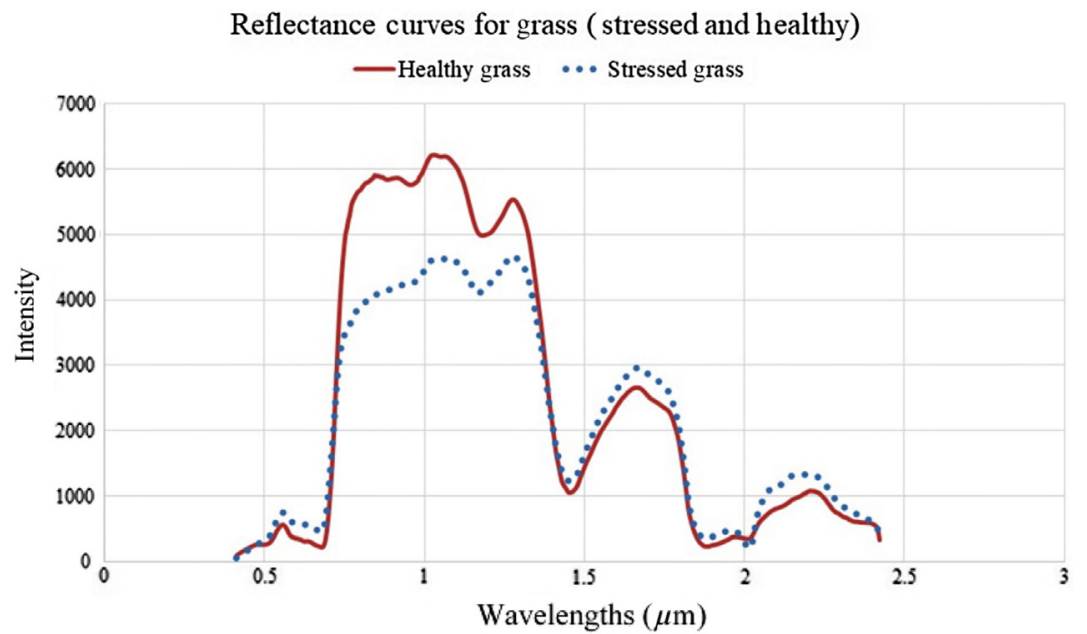

(a)

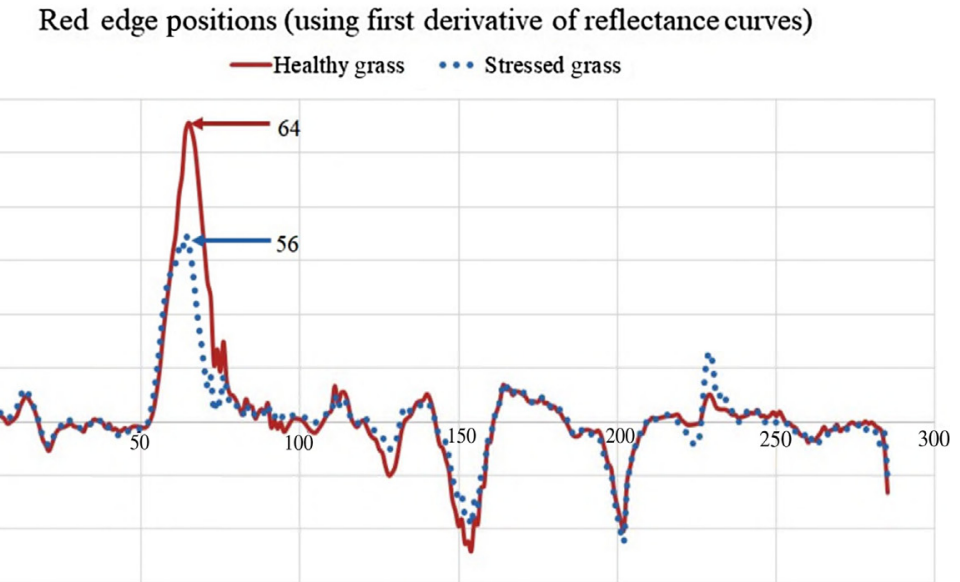

Band numbers

(b)

Fig. 6 The near-infrared plateau is a measure of the chlorophyll concentration, and its decline, in the leaf. This concentration is indicative of the health of the plant. (a) The comparison of the intensity curves of Healthy Grass and Stressed Grass shows the variation of the plateau, indicating that the chlorophyll concentration in the leaf declines under stress. (b) The REP shifts toward the red wavelengths when the amount of chlorophyll in vegetation declines. The shift is indicative of the health of the vegetation, grass in this case.

the constraints of other classes. ${ }^{8}$ In the case of PCM, the membership function of each class is defined using the following constraints [Eqs. (6)-(8) ${ }^{8,60}$ ]:

$$
\begin{gathered}
u_{i j} \in[0,1] \text { for all } i \text { and } j, \\
0<\sum_{j=1}^{n} u_{i j} \leq N \text { for all } i, \\
\max _{i} u_{i j}>0 \text { for all } j,
\end{gathered}
$$

where $u_{i j}$ is the membership value of pixel $x_{i}$ belonging to a class $\beta_{i}$ and $N$ is the number of pixels (or feature points).

The classification was performed using an in-house tool developed by the coauthors. The tool accepts an input of a dataset, converts the original intensity values to reflectance data (the VNIR 
and SWIR data are encoded in 14-bit and 13-bit formats), and allows a click-selection of pixels as input to the supervised classification method. The generated output is individual grayscale images per class. The value, between 0 and 255, defines the class percentage of each pixel. An additional image is output, containing information of all the classes without a strong membership function into the input classes.

\subsection{Spectral Indices}

Spectral inferences about the classes were made using identification "keys" or specific bands that allow the classifier to distinguish between targets. These keys are contrasting spectral behavior of absorption and reflection (troughs and peaks) in the spectral curve. The spectral properties of all the identified classes were compared. Table 1 shows the identifiable properties and their corresponding wavelength regions for the vegetation features. Using this method of feature selection, we reduced the dimensionality of the hyperspectral dataset to bands with the most appropriate information, so we consider this to be a knowledge-based dimensionality reduction approach. The bands selected in this process form the input database for the classifier.

Following the identification of keys with the necessary spectral information, spectral indices were defined using band combinations (Table 2). The indices did not always uniquely identify a specific class, e.g., the increase in reflectance for Artificial Turf and Roof spectral curves [indicated in Fig. 5(d)]. Therefore, when spectral indices are defined in these regions, both classes are highlighted. Such conflicts among the 20 classes considered in this study are shown in Table 3.

Table 1 Correlations between wavelength regions and their biological significance. ${ }^{61-63}$

\begin{tabular}{|c|c|c|}
\hline $\begin{array}{l}\text { Wavelength } \\
\text { region }\end{array}$ & $\begin{array}{l}\text { Wavelength } \\
(\lambda)(\mu \mathrm{m})\end{array}$ & Band description and significance \\
\hline Blue & 0.49 & $\begin{array}{l}\text { Sensitive to loss of chlorophyll, browning, ripening, senescence, and soil } \\
\text { background effects. Also sensitive to carotenoid pigments. }\end{array}$ \\
\hline Green 1 & 0.52 & $\begin{array}{l}\text { Maximum "positive change in reflectance per unit variation in wavelength" } \\
\text { of visible spectrum is seen around this green wavelength and is sensitive } \\
\text { to pigment content }\end{array}$ \\
\hline Green 2 & 0.55 & $\begin{array}{l}\text { Green peak in the visible spectrum; strongly related to chlorophyll } \\
\text { content. }\end{array}$ \\
\hline Green 3 & 0.575 & $\begin{array}{l}\text { Maximum "negative change in reflectance per unit variation in wavelength" } \\
\text { of visible spectrum is seen around this green wavelength and is sensitive } \\
\text { to pigment content. }\end{array}$ \\
\hline Red 1 & 0.66 & Chlorophyll absorption pre-maxima (reflectance minima-1) \\
\hline Red 2 & 0.675 & $\begin{array}{l}\text { Chlorophyll absorption maxima. Greatest soil-crop contrast seen at this } \\
\text { wavelength. }\end{array}$ \\
\hline Red edge-1 & 0.7 & $\begin{array}{l}\text { Chlorophyll absorption postmaxima (reflectance minima-2). This point } \\
\text { marks the change of maximum red-absorption to dramatic increase in red } \\
\text { reflectance along the red edge. This has been found to be sensitive to } \\
\text { stress levels in vegetation. }\end{array}$ \\
\hline Red edge-2 & 0.72 & $\begin{array}{l}\text { Critical point on the red edge where the "maximum change of slope } \\
\text { reflectance spectra per unit change in wavelength" occurs. Sensitive } \\
\text { to temporal changes in crop growth, stress, etc. }\end{array}$ \\
\hline NIR & 0.845 & Center of the "NIR shoulder." Strongly correlated to chlorophyll. \\
\hline NIR peak-1 & 0.905 & $\begin{array}{l}\text { Peak of the NIR spectrum. Sensitive to stress or growth stages of some } \\
\text { crops, where there is a significant change in reflectance along the NIR } \\
\text { shoulder. Useful for calculating crop moisture sensitive index. }\end{array}$ \\
\hline NIR peak-2 & 0.920 & Peak of the NIR spectrum \\
\hline $\begin{array}{l}\text { NIR-moisture } \\
\text { sensitive }\end{array}$ & 0.975 & $\begin{array}{l}\text { Center of moisture-sensitive portion of NIR. Various measures of plant } \\
\text { moisture can be made from this wavelength's reflectance. }\end{array}$ \\
\hline
\end{tabular}


Table 2 Spectral indices database chosen for classification input; 20 classes are identified in these indices, as some indices identify $>11$ class. Note that the band numbers correspond to APEX OSD data.

\begin{tabular}{lcc}
\hline \hline Object class & Formulated/applied index & APEX band combination \\
\hline Artificial turf & Band ratio & $95,76(0.8752 \mu \mathrm{m}, 0.7716 \mu \mathrm{m})$ \\
Black roof & Band ratio & $160,149(1.45 \mu \mathrm{m}, 1.343 \mu \mathrm{m})$ \\
Buildings & NDBI & $160,145(1.45 \mu \mathrm{m}, 1.304 \mu \mathrm{m})$ \\
Clay soil & Band ratio & $236,225(2.09 \mu \mathrm{m}, 2.007 \mu \mathrm{m})$ \\
Coniferous forest & Band ratio & $85,53(0.8167 \mu \mathrm{m}, 0.6816 \mu \mathrm{m})$ \\
Deciduous forest & MTVI1 & $81,17,52(0.7958 \mu \mathrm{m}, 0.5567 \mu \mathrm{m}, 6784 \mu \mathrm{m})$ \\
Red synthetic ground & Band ratio & $197,192(1.782 \mu \mathrm{m}, 1.74 \mu \mathrm{m})$ \\
Stressed grass & Band ratio & $234,226(2.074 \mu \mathrm{m}, 2.015 \mu \mathrm{m})$ \\
Vineyard & Modified NDVI (devised & $236,225(2.09 \mu \mathrm{m}, 2.007 \mu \mathrm{m})$ \\
& in SWIR) & \\
Water & NDectral curve behavior & $183,146(1.662 \mu \mathrm{m}, 1.314 \mu \mathrm{m})$ \\
Roof & Band ratio & $142,122(1.275 \mu \mathrm{m}, 1.082 \mu \mathrm{m})$ \\
Basic vegetation index & MSAVI & $85,53(0.8167 \mu \mathrm{m}, 0.6816 \mu \mathrm{m})$ \\
\hline \hline
\end{tabular}

Table 3 Conflicting classes from identified spectral keys.

\begin{tabular}{lc}
\hline \hline Object class-specific index & Roof \\
\hline Artificial turf & Water \\
Black roof & Multiple classes \\
Buildings & Artificial turf, vineyards \\
Clay soil & Grass \\
Deciduous forest & Multiple classes \\
Red synthetic ground & Clay soil, vineyard \\
Stressed grass & Clay soil, stressed grass \\
Vineyard & All vegetation in the study area \\
Basic vegetation index
\end{tabular}

\subsection{Spatial Indices from Texture Analysis}

The class-specific feature extraction of the spectral characteristics provided us with the second input database (the first being the input of principal components ${ }^{6}$ ) for the classifier. However, when dealing with classes that have comparable spectral behavior (e.g., forests and grass), spatial distribution would act as a distinguishing factor. Therefore, texture analysis (neighborhood distribution) was considered for the purpose of exploiting the spatial distribution of the classes.

Gray-level co-occurrence matrix texture analysis has been described in detail. ${ }^{21}$ The texture measurement involves the selection of a neighborhood of pixels followed by quantifying the gray-level variation to identify the most appropriate textural feature. The analysis is performed on the spectral indices output to distinguish between spectrally similar targets. The choice of neighborhood for the pixels is dependent on the spatial resolution of the image data and the 
extent of the target. Smaller targets that span a few pixels require smaller windows of texture analysis, whereas larger targets benefit from the flexibility of larger spectral windows. After considering the available texture measures, we chose the following [Eqs. (9)-(11)]:

$$
\begin{gathered}
\operatorname{Mean}\left(\mu_{i}\right)=\sum_{i, j=0}^{N-1} i\left(P_{i, j}\right) ; \quad \operatorname{Mean}\left(\mu_{j}\right)=\sum_{i, j=0}^{N-1} j\left(P_{i, j}\right), \\
\text { Entropy }=\sum_{i, j=0}^{N-1} P_{i, j}\left(-\ln P_{i, j}\right), \\
\text { Second Moment }=\sum_{i, j=0}^{N-1}\left(P_{i, j}\right)^{2},
\end{gathered}
$$

where $P_{i, j}$ is the $(i, j)^{\prime}$ th entry in the gray tone spatial dependence matrix and $N$ is the number of gray levels in the quantized image.

We initially tested multiple window sizes and choice of the neighborhood was dependent on the spatial extent of the classes. Thus, classes such as Roof did not need window sizes larger than $3 \times 3$. Due to variations in the extent of vegetation across the image, larger window-sizes could be accommodated and provide better results. The chosen spatial indices (Table 4) were added to the spectral indices to make up the third input for classification.

Second moment generates better results when a few pixels in the neighborhood are higher in intensity and, therefore, could improve the identification and classification accuracy of vegetation classes.

Entropy generates high output values when measured on neighborhoods that have continuous and consistent pixel values. The texture measure could derive the desired output when applied to features, such as black roof, which have a consistent distribution of pixel values. The mean distribution of pixel values highlights pixels of high intensity and increases the separability of classes, such as roof and clay soil.

The spectral indices input database and the combined spectral and spatial indices database was evaluated for classification accuracy using the PCM classifier and the results were compared to determine the suitability of the method. The primary motivation for this research was to better understand the implications of combining spectral and spatial information for the classification of the dataset, following entropy analysis and accuracy assessment after defuzzification. ${ }^{6}$ The defuzzification of the classification outputs assigns a class label of the highest membership to a considered pixel. Although a few classes were not identified by the PCM classifier, their results were measured after defuzzification through which every pixel was assigned a class label. The accuracy assessment includes:

1. User's accuracy (UA), which is the reliability or probability that a pixel class on the image represents the same on the ground.

2. Producer's accuracy (PA), which represents how well a certain area has been classified.

3. Overall accuracy $(\mathrm{OA})$, which represents the number of pixels correctly classified when compared with ground truth and knowledge information.

Table 4 Selected texture measures (spatial indices) for input to the classifier.

\begin{tabular}{lcc}
\hline \hline Source index & Texture & Window size (neighborhood) \\
\hline Black roof & Entropy & $3 \times 3$ window \\
Clay soil & Mean & $3 \times 3$ window \\
Coniferous forest & Second moment & $3 \times 3$ window \\
MSAVI vegetation index & Second moment & $5 \times 5$ window \\
Roof & Mean & $3 \times 3$ window \\
\hline \hline
\end{tabular}




\section{Results}

To briefly review, the classification involved input of the dataset to the in-house tool. Input to the supervised classification process was given as input from the user. This input was used to construct spectral signatures of classes and used to identify similar pixels in the dataset. The output of the classification process is individual grayscale images, one per input class. An additional grayscale output contains all the unclassified information, as is the unconstrained nature of the membership functions in the possibilistic $c$-means algorithm.

We selected the 12 spectral and five spatial input bands. Combinations of these were used to create the input database for classification. The results are presented in the order of baseline classification, followed by spectral indices alone, followed by a combination of texture features in addition to the spectral indices input. Entropy measures were not calculated in scenarios where classes were not identified and are shown using the symbol "/" in Tables 5-7.

The first inputs to the classifier were the principal components derived by PCA. The appropriate number of principal components was determined by entropy analysis. ${ }^{6}$ The classification

Table 5 Entropy determination and classification results when using the optimally chosen principal components (seven PCs) and only the spectral indices as input for the classifier. The symbol "p" indicates that the class was not identified in the classification process.

\begin{tabular}{|c|c|c|c|c|c|c|}
\hline \multirow[b]{2}{*}{ Class } & \multicolumn{3}{|c|}{ PC input database (7 features) } & \multicolumn{3}{|c|}{$\begin{array}{l}\text { Indices input database } \\
\text { (12 features) }\end{array}$} \\
\hline & Entropy & UA & PA & Entropy & UA & PA \\
\hline Artificial turf & / & 20 & 100 & 1.94 & 100 & 100 \\
\hline Black roof & I & 62.5 & 83.34 & l & 55.56 & 100 \\
\hline Building & 1.35 & 100 & 50 & l & 100 & 42.86 \\
\hline Clay soil & 1.334 & 88.89 & 80 & 1.45 & 90 & 100 \\
\hline Grass & I & 40 & 44.45 & l & 40 & 57.14 \\
\hline Lawn tennis court & 1.26 & 70 & 100 & l & 40 & 100 \\
\hline Coniferous forest & 1.3 & 60 & 40 & 1.62 & 80 & 57.14 \\
\hline Deciduous forest & I & 80 & 29.63 & 1.6 & 90 & 50 \\
\hline Pasture & I & 30 & 100 & 1.46 & 55.56 & 100 \\
\hline Railway & I & 28.57 & 100 & I & 30 & 50 \\
\hline Red roof & 1.1 & 100 & 25 & l & 66.67 & 54.55 \\
\hline Red synthetic ground & 1.15 & 60 & 85.71 & l & 60 & 100 \\
\hline Road & 0.86 & 90 & 47.37 & I & 70 & 23.34 \\
\hline Roof & I & 40 & 50 & 0.68 & 100 & 66.67 \\
\hline Sand & 1.23 & 50 & 100 & l & 10 & 100 \\
\hline Stressed grass & I & 40 & 40 & 1.49 & 70 & 70 \\
\hline Synthetic sports surface & l & 100 & 100 & l & 44.45 & 100 \\
\hline Vineyard & 1.07 & 30 & 50 & 1.49 & 66.67 & 75 \\
\hline Water & I & 100 & 90.9 & 2.06 & 100 & 90.91 \\
\hline Yellow tartan & I & 33.34 & 100 & 0.6 & 30 & 100 \\
\hline $\begin{array}{l}\text { Overall classification } \\
\text { accuracy (\%) }\end{array}$ & & 59.50 & & & 65 & \\
\hline
\end{tabular}

Note: The bold values are the final results at each step. 
Table 6 Entropy determination and classification results when using the indices and textures database (17 and 16 features) as input for the classifier. The symbol " $"$ indicates that the class was not identified in the classification process.

\begin{tabular}{|c|c|c|c|c|c|c|}
\hline \multirow[b]{2}{*}{ Class } & \multicolumn{3}{|c|}{ Indices and textures (17 features) } & \multicolumn{3}{|c|}{$\begin{array}{l}\text { Indices and textures without } \\
\text { MSAVI-based textures } \\
\text { (16 features) }\end{array}$} \\
\hline & Entropy & UA & PA & Entropy & UA & PA \\
\hline Artificial turf & 1.146 & 80 & 80 & 1.274 & 100 & 100 \\
\hline Black roof & l & 37.5 & 75 & l & 12.5 & 100 \\
\hline Building & 0.63 & 60 & 31.58 & 0.44 & 100 & 63.63 \\
\hline Clay soil & 1.212 & 80 & 100 & 1.2 & 100 & 100 \\
\hline Grass & 1.004 & 60 & 85.71 & 1.25 & 90 & 90 \\
\hline Lawn tennis court & 0.64 & 80 & 66.67 & 0.5 & 88.89 & 100 \\
\hline Coniferous forest & 0.956 & 100 & 71.43 & 1.34 & 100 & 83.33 \\
\hline Deciduous forest & 1.02 & 80 & 61.54 & 1.35 & 100 & 76.92 \\
\hline Pasture & 0.966 & 71.43 & 83.34 & 1.25 & 44.44 & 100 \\
\hline Railway & l & 10 & 33.34 & I & 25 & 100 \\
\hline Red roof & l & 20 & 100 & 0.46 & 100 & 91.67 \\
\hline Red synthetic ground & 0.65 & 81.82 & 90 & 0.55 & 42.86 & 100 \\
\hline Road & l & 50 & 15.625 & 0.44 & 88.89 & 24.24 \\
\hline Roof & l & 30 & 27.27 & l & 71.43 & 75 \\
\hline Sand & l & 20 & 33.34 & I & 14.29 & 100 \\
\hline Stressed grass & 1.126 & 80 & 80 & 1.3 & 87.5 & 100 \\
\hline Synthetic sports surface & 0.65 & 50 & 100 & 0.55 & 87.5 & 100 \\
\hline Vineyard & 0.94 & 80 & 88.89 & 1.1 & 90.91 & 83.34 \\
\hline Water & 1.44 & 100 & 76.92 & 1.85 & 90 & 75 \\
\hline Yellow tartan & I & 10 & 100 & 0.5 & 33.33 & 100 \\
\hline $\begin{array}{l}\text { Overall classification } \\
\text { accuracy }(\%)\end{array}$ & & $62.50 \%$ & & & $80.50 \%$ & \\
\hline
\end{tabular}

Note: The bold values are the final results at each step.

using these principal components yielded an overall accuracy of $59.50 \%$. The second input to the classifier was the spectral indices database (12 features shown in Table 2). As well as improving the overall classification accuracy to $65 \%$ (Table 5), the spectral indices input database also identified seven classes that were previously unidentified when using the principal components alone. Entropy measures were calculated for an average of 50 membership vectors per class.

When comparing the indices input (Table 5) and the spectral-spatial indices (Table 6) for classification, we observed a decrease in entropy measures for the majority of the classes, inferring a reduction in the level of uncertainty when applying class labels to the pixels. Although the entropy was lower, the defuzzified outputs also achieved a lower classification accuracy of $62.50 \%$. However, a significant improvement in classification accuracy was achieved for vegetation classes, such as vineyard (Table 6).

Although the addition of spatial indices improved the classification accuracy for specific individual classes (e.g., synthetic sports surface, pastures), the overall classification accuracy dropped below the $65 \%$ achieved when using spectral indices alone. Therefore, the assignment 
Table 7 Entropy determination and classification results when using the indices and textures database (15 features) as input for the classifier. The symbol "p" indicates that the class was not identified during the classification process.

\begin{tabular}{|c|c|c|c|}
\hline \multirow[b]{2}{*}{ Class } & \multicolumn{3}{|c|}{$\begin{array}{l}\text { Indices and textures without MSAVI-based and } \\
\text { black roof entropy (15 features) }\end{array}$} \\
\hline & Entropy & UA & PA \\
\hline Artificial turf & 1.296 & 100 & 100 \\
\hline Black roof & l & 30 & 100 \\
\hline Building & 0.399 & 100 & 70.59 \\
\hline Clay soil & 1.14 & 100 & 100 \\
\hline Grass & 1.17 & 80 & 80 \\
\hline Lawn tennis court & 0.46 & 75 & 100 \\
\hline Coniferous forest & 1.18 & 100 & 83.33 \\
\hline Deciduous forest & 1.2 & 90 & 81.82 \\
\hline Pasture & 1.19 & 70 & 100 \\
\hline Railway & l & 12.5 & 33.33 \\
\hline Red roof & 0.44 & 63.64 & 70 \\
\hline Red synthetic ground & 0.52 & 75 & 85.71 \\
\hline Road & 0.41 & 73.33 & 28.95 \\
\hline Roof & l & 72.73 & 66.67 \\
\hline Sand & 1 & 0 & 0 \\
\hline Stressed grass & 1.16 & 100 & 63.64 \\
\hline Synthetic sports surface & 0.54 & 75 & 100 \\
\hline Vineyard & 1.1 & 50 & 100 \\
\hline Water & 1.138 & 100 & 83.33 \\
\hline Yellow tartan & 0.5 & 11.12 & 100 \\
\hline Overall classification accuracy (\%) & & 72 & \\
\hline
\end{tabular}

Note: The bold values are the final results at each step.

of spatial classes was investigated while using a total of 16 indices (without MSAVI-based texture) and 15 (without MSAVI-based and black roof entropy textures) while including all spectral indices (Table 2). The combination of 16 indices achieved the highest accuracy of $80.50 \%$ (Table 6) with better individual accuracies and lower degrees of uncertainty for classification.

To test the significance of the remaining four spatial indices, various combinations of usage were tested. The only significant result was achieved when using 15 features (excluding MSAVIbased second moment and black roof entropy texture measures), which achieved an overall classification accuracy of $72 \%$.

\section{Discussions}

Within the scope of this study and the attributes of the dataset, our results confirm that using spectral-spatial indices can improve the accuracy of classification. This combination of knowledge-based feature selection and extraction for classification improves classification accuracy for 
specific classes while intrinsically reducing the dimensionality of the hyperspectral dataset by choosing the information input to the classifier.

However, a key inference from the results is that not all spatial indices added valuable information to the database. For example, the removal of MSAVI-based texture improved the overall classification accuracy of the dataset. We initially considered the second moment of the MSAVI spectral index as an input to distinguish the vegetation classes based on their spectral response and spatial distribution. However, the removal of this spatial index greatly improved the overall classification accuracy. We consider, retrospectively, that the addition of the index may have reduced the variation between the vegetation classes. The evidence of this can be found in the reduction of accuracies while comparing Coniferous Forest and Deciduous Forest in Table 6.

The baseline classification used a dimensionality-reduced input from the PCA to reduce the hyperspectral data to its intrinsic dimensionality of seven principal components. The lowest number of classes was identified and a subsequent classification achieved an accuracy of $59.50 \%$. The usage of spectral indices (Table 2), irrespective of the arising conflicts of classes (Table 3), improved the classification accuracy for the dataset. The knowledge-based selection of spectral information and the reduction of dimensionality using indices allowed the classifier to identify and classify the pixels with greater accuracy. The use of this spectral information improved the classification of vegetation classes (coniferous forest, deciduous forest, pasture, and stressed grass). Marginal improvement was also observed for the classification of clay soil and artificial turf.

Classes such as black roof, grass, and synthetic sports surface remained unidentified when only the spectral information was used. Furthermore, the railway class remained unclassified for all the spectral-spatial indices inputs. Due to its nature, the railway network is better identified using spatial information, so object-oriented classification approaches may be more suitable for the classification of railway networks.

The identification of classes improved when spectral and spatial features were combined as the input database. Although the overall accuracy was lower when compared with the spectral indices input, the accuracy of classifying grass, coniferous forest, and deciduous forest improved. The entropy measure was substantially reduced in this case, in agreement with better classification results and lower degrees of uncertainty when assigning class labels. Further investigation is required to identify the spatial features that add most value against dimensionality, thereby formulating the next input database of 12 spectral and 4 spatial indices (excluding the MSAVI-based texture). In comparison with the database of 17 input features (Table 6), entropy measures for classes such as buildings, clay soil, lawn tennis court, red synthetic ground, and synthetic sports surface were lower than the input database of 17 features. For the same classes, there was also an improvement in classification accuracy (UA). The road class was identified when the input features were reduced from 17 to 16, also with a low entropy and high (UA) accuracy. Some of the classes (vineyard and forest classes) saw an increase in entropy but also an increase in classification accuracy. Conversely, the Water class showed an increase in entropy and a decrease in classification accuracy. The MSAVI-based texture index formulated from the MSAVI spectral index was initially added to the input database to distinguish between vegetation classes. However, the overall classification accuracy is the best of the many combinations of inputs to the classifier, thereby confirming the value of the remaining texture indices (Table 4). The second moment spatial index, derived from coniferous forest spectral index, provided information sufficient to distinguish the grass and forest classes and to simultaneously improve their classification accuracy. Only four classes could not be identified using PCM and the 16 feature input database, and an overall improvement of accuracy was achieved.

\section{Conclusions and Future Work}

Our results show that spectral and spatial indices can be combined for classification. The use of spectral and spatial information improves the accuracy of the classifier, but the input dataset must be evaluated to find the best possible combination of indices. We find that every dataset is unique, and the assessment of knowledge-based dimensionality reduction requires an understanding of the specific class that needs to be identified. 
Future research could proceed in the following directions:

1. Comparison with other dimensionality reduction methods to see how they fare against the methodology in this literature.

2. Evaluating the application of object-oriented classification because it accommodates both spectral and spatial information.

3. A few of the classes were not classified regardless of the combination of inputs for the classifier. Although this has been attributed to the smaller spatial distribution of pixels corresponding to these classes, their contribution to this classification approach could be evaluated by considering a different study area that has a higher distribution of such classes.

4. The optimal choice of hyperspectral bands is an ongoing domain of research, e.g., the selection of optimal bands for agricultural crop characteristics using field-collected biophysical variables. ${ }^{61}$ For specific classes, an automated method that identifies optimal bands could be developed.

\section{Acknowledgments}

We would like to thank Dr. Sc.Nat. Andreas Hueni (University of Zurich) for his assistance during the analysis of the imagery and the related ground-truth information. We would also like to thank Dr. Richard Twyman for his assistance in the drafting and presentation of the paper. The current paper is adopted and improved from Kallepalli et al. 2016. ${ }^{64}$

\section{References}

1. S.-E. Qian and G. Chen, "A new nonlinear dimensionality reduction method with application to hyperspectral image analysis," in IEEE Int. Geoscience and Remote Sensing Symp., IEEE, pp. 270-273 (2007).

2. T. M. Lillesand, R. W. Kiefer, and J. W. Chipman, Remote Sensing and Image Interpretation, 5th edn., John Wiley and Sons (Asia) Pvt. Ltd., Singapore and Sanjeev Offset Printers, Delhi (2007).

3. C. R. da Silva, J. A. S. Centeno, and S. R. Aranha, "Reduction of the dimensionality of hyperspectral data for the classification of agricultural scenes," in 13th Symp. Deformation Measurements and Analysis (2008).

4. G. Vane and A. F. Goetz, "Terrestrial imaging spectroscopy," Remote Sens. Environ. 24(1), 1-29 (1988).

5. G. Hughes, "On the mean accuracy of statistical pattern recognizers," IEEE Trans. Inf. Theory 14, 55-63 (1968).

6. A. Kallepalli, A. Kumar, and K. Khoshelham, "Entropy based determination of optimal principal components of Airborne Prism EXperiment (APEX) imaging spectrometer data for improved land cover classification," Int. Arch. Photogramm. Remote Sens. Spat. Inf. Sci. XL-8(8), 781-786 (2014).

7. J. C. Harsanyi and C.-I. Chang, "Hyperspectral image classification and dimensionality reduction: an orthogonal subspace projection approach," IEEE Trans. Geosci. Remote Sens. 32(4), 779-785 (1994).

8. R. Krishnapuram and J. M. Keller, "A Possibilistic approach to clustering," IEEE Trans. Fuzzy Syst. 1(2), 98-110 (1993).

9. A. Kallepalli, "Spectral and spatial indices based specific class identification from Airborne Hyperspectral data," in Master's Thesis, Faculty of Geo-Information Science and Earth Observation (ITC), University of Twente (2014).

10. A. Swatantran et al., "Mapping biomass and stress in the Sierra Nevada using lidar and hyperspectral data fusion," Remote Sens. Environ. 115, 2917-2930 (2011).

11. M. A. Cochrane, "Using vegetation reflectance variability for species level classification of hyperspectral data," Int. J. Remote Sens. 21(10), 2075-2087 (2010).

12. G. Lu and B. Fei, "Medical hyperspectral imaging: a review," J. Biomed. Opt. 19(1), 010901 (2014). 
13. J. M. Van Campenhout, "On the peaking of the Hughes mean recognition accuracy: the resolution of an apparent paradox," IEEE Trans. Syst. Man Cybern. 8(5), 390-395 (1978).

14. A. Jain and D. Zongker, "Feature selection: evaluation, application, and small sample performance," IEEE Trans. Pattern Anal. Mach. Intell. 19(2), 153-158 (1997).

15. I. T. Jolliffe, Principal Component Analysis, Springer, New York (2002).

16. C. Rodarmel and J. Shan, "Principal component analysis for hyperspectral image classification," Surv. Land Inf. Sci. 62(2), 115-122 (2002).

17. A. Gillespie et al., "Interpretation of residual images: spectral mixture analysis of AVIRIS images, Owens Valley, California," in Proc. 2nd Airborne Visible/Infrared Imaging Spectrometer (AVIRIS) Workshop, NASA Jet Propulsion Laboratory Pasadena, California, pp. 243-270 (1990).

18. S. B. Serpico and G. Moser, "Extraction of spectral channels from hyperspectral images for classification purposes," IEEE Trans. Geosci. Remote Sens. 45, 484-495 (2007).

19. A. Plaza et al., "Dimensionality reduction and classification of hyperspectral image data using sequences of extended morphological transformations," IEEE Trans. Geosci. Remote Sens. 43, 466-479 (2005).

20. J. R. Jensen, "Biophysical remote sensing," Ann. Assoc. Am. Geogr. 73(1), 111-132 (1983).

21. R. M. Haralick et al., "Textural features for image classification," IEEE Trans. Syst. Man Cybern. SMC-3(6), 610-621 (1973).

22. J. Estes, E. Hajic, and L. R. Tinney, "Fundamentals of image analysis: analysis of visible and thermal infrared data," in Manual of Remote Sensing, R. Colwell, Ed., Vol. 1, pp. 987-1124, American Society of Photogrammetry, Falls Church, Virginia (1983).

23. P. R. Wolf and B. A. Dewitt, Elements of Photogrammetry: With Applications in GIS, Vol. 3, McGraw-Hill, New York (2000).

24. C. F. Jordan, "Derivation of leaf-area index from quality of light on the forest floor," Ecology 50(4), 663-666 (1969).

25. S. Keyworth, M. Jarman, and K. Medcalf, "Assessing the extent and severity of erosion on the upland organic soils of Scotland using earth observation-a GIFTSS implementation test," Tech. Rep., Environment Systems Limited (2009).

26. G.-R. Liu et al., "Comparison of the NDVI, ARVI and AFRI vegetation index, along with their relations with the AOD using SPOT 4 vegetation data," Terr. Atmos. Oceanic Sci. 15(1), 15-32 (2004).

27. J. W. Rouse, Jr. et al., "Monitoring vegetation systems in the great plains with ERTS," in Third Earth Resources Technology Satellite-1 Symp., National Aeronautics and Space Administration, Washington DC, NASA SP-351, Vol. 1, pp. 309-317 (1973).

28. A. R. Huete, "A soil-adjusted vegetation index (SAVI)," Remote Sens. Environ. 25(3), 295 309 (1988).

29. G. A. Carter, W. G. Cibula, and T. R. Dell, "Spectral reflectance characteristics and digital imagery of a pine needle blight in the south-eastern United States," Can. J. For. Res. 26(3), 402-407 (1996).

30. J. Vogelmann, B. Rock, and D. Moss, "Red edge spectral measurements from sugar maple leaves," Remote Sens. 14(8), 1563-1575 (1993).

31. A. A. Gitelson and M. N. Merzlyak, "Remote estimation of chlorophyll content in higher plant leaves," Int. J. Remote Sens. 18(12), 2691-2697 (1997).

32. D. Haboudane et al., "Hyperspectral vegetation indices and novel algorithms for predicting green LAI of crop canopies: modeling and validation in the context of precision agriculture," Remote Sens. Environ. 90(3), 337-352 (2004).

33. N. H. Broge and E. Leblanc, "Comparing prediction power and stability of broadband and hyperspectral vegetation indices for estimation of green leaf area index and canopy chlorophyll density," Remote Sens. Environ. 76(2), 156-172 (2001).

34. C. Daughtry et al., "Estimating corn leaf chlorophyll concentration from leaf and canopy reflectance," Remote Sens. Environ. 74(2), 229-239 (2000).

35. J. Qi et al., "A modified soil adjusted vegetation index," Remote Sens. Environ. 48(2), 119 126 (1994).

36. C. D. Elvidge and Z. Chen, "Comparison of broad-band and narrow-band red and near-infrared vegetation indices," Remote Sens. Environ. 54(1), 38-48 (1995). 
37. C. J. Tucker, "Red and photographic infrared linear combinations for monitoring vegetation," Remote Sens. Environ. 8(2), 127-150 (1979).

38. A. J. Richardson and C. Wiegand, "Distinguishing vegetation from soil background information [by gray mapping of Landsat MSS data]," Photogramm. Eng. Remote Sens. 43, 1541-1552 (1977).

39. M. S. Kim, "The use of narrow spectral bands for improving remote sensing estimations of fractionally absorbed photosynthetically active radiation (fAPAR)," Master's Thesis, Department of Geography, University of Maryland, College Park, Maryland (1994).

40. P. J. Zarco-Tejada et al., "Scaling-up and model inversion methods with narrowband optical indices for chlorophyll content estimation in closed forest canopies with hyperspectral data," IEEE Trans. Geosci. Remote Sens. 39(7), 1491-1507 (2001).

41. J. Peñuelas et al., "Reflectance indices associated with physiological changes in nitrogen-and water-limited sunflower leaves," Remote Sens. Environ. 48(2), 135-146 (1994).

42. P. J. Zarco-Tejada et al., "Hyperspectral indices and model simulation for chlorophyll estimation in open-canopy tree crops," Remote Sens. Environ. 90(4), 463-476 (2004).

43. P. J. Zarco-Tejada et al., "Assessing vineyard condition with hyperspectral indices: leaf and canopy reflectance simulation in a row-structured discontinuous canopy," Remote Sens. Environ. 99(3), 271-287 (2005).

44. A. Hall et al., "Optical remote sensing applications in viticulture-a review," Aust. J. Grape Wine Res. 8(1), 36-47 (2002).

45. J. Gamon, J. Penuelas, and C. Field, "A narrow-waveband spectral index that tracks diurnal changes in photosynthetic efficiency," Remote Sens. Environ. 41(1), 35-44 (1992).

46. A. A. Gitelson et al., "Assessing carotenoid content in plant leaves with reflectance spectroscopy," Photochem. Photobiol. 75(3), 272-281 (2002).

47. Y. Zha, J. Gao, and S. Ni, "Use of normalized difference built-up index in automatically mapping urban areas from TM imagery," Int. J. Remote Sens. 24(3), 583-594 (2003).

48. B.-C. Gao, "NDWI-a normalized difference water index for remote sensing of vegetation liquid water from space," Remote Sens. Environ. 58(3), 257-266 (1996).

49. M. Herold, H. Couclelis, and K. C. Clarke, "The role of spatial metrics in the analysis and modeling of urban land use change," Comput. Environ. Urban Syst. 29, 369-399 (2005).

50. D. A. Clausi, "An analysis of co-occurrence texture statistics as a function of grey level quantization," Can. J. Remote Sens. 28(1), 45-62 (2002).

51. B. Datt et al., "Preprocessing EO-1 hyperion hyperspectral data to support the application of agricultural indexes," IEEE Trans. Geosci. Remote Sens. 41, 1246-1259 (2003).

52. K. I. Itten et al., "APEX - the hyperspectral ESA airborne prism experiment," Sensors 8, 6235-6259 (2008).

53. D. Schlapfer et al., "Calibration and validation concept for the Airborne Prism EXperiment (APEX)," Can. J. Remote Sens. 26(5), 455-465 (2000).

54. A. Hueni et al., "Structure, components, and interfaces of the Airborne Prism EXperiment (APEX) processing and archiving facility," IEEE Trans. Geosci. Remote Sens. 47(1), 29-43 (2009).

55. M. Jehle et al., "APEX-current status, performance and validation concept," in IEEE SENSORS, pp. 533-537 (2010).

56. "Free data cubes-APEX - airborne prism experiment," http://www.apex-esa.org/content/ free-data-cubes (6 August 2016).

57. "APEX open science data set leaflet" (2011).

58. "SwissTopo web portal," http://map.geo.admin.ch/?topic=ech\&lang=en\&X=257116.37\& $\mathrm{Y}=664823.41 \&$ zoom=6\&bgLayer=ch.swisstopo.swissimage (8 August 2016).

59. M. A. Cho and A. K. Skidmore, "A new technique for extracting the red edge position from hyperspectral data: the linear extrapolation method," Remote Sens. Environ. 101(2), 181-193 (2006).

60. G. M. Foody, "Sub-pixel methods in remote sensing," in Remote Sensing Image Analysis: Including the Spatial Domain, S. M. de Jong and F. D. van der Meer, Eds., pp. 37-49, Kluwer Academic Publishers, Dordrecht (2004). 
61. P. S. Thenkabail, R. B. Smith, and E. De Pauw, "Evaluation of narrowband and broadband vegetation indices for determining optimal hyperspectral wavebands for agricultural crop characterization," Photogramm. Eng. Remote Sens. 68(6), 607-622 (2002).

62. P. S. Thenkabail, R. B. Smith, and E. De Pauw, "Hyperspectral vegetation indices and their relationships with agricultural crop characteristics," Remote Sens. Environ. 71(2), 158-182 (2000).

63. J. Penuelas et al., "The reflectance at the $950-970 \mathrm{~nm}$ region as an indicator of plant water status," Int. J. Remote Sens. 14(10), 1887-1905 (1993).

64. A. Kallepalli et al., "Application of spectral and spatial indices for specific class identification in Airborne Prism EXperiment (APEX) imaging spectrometer data for improved land cover classification," Proc. SPIE 10005, 100050Z (2016).

Akhil Kallepalli is a research student currently pursuing his $\mathrm{PhD}$ in optical imaging and laser systems applications with the Centre for Electronics Warfare, Cranfield University, based in Shrivenham, UK, at the Defence Academy of the UK. He received his BTech degree from Andhra University (2012) and his MSc degree through a joint program between Indian Institute of Remote Sensing and ITC, University of Twente (2014). His current research interests include biomedical applications of lasers, hyperspectral image processing, and coastal change detection. He is a current student member of SPIE.

Anil Kumar is a scientist/engineer with the Indian Institute of Remote Sensing, Dehradun, India. He received his BTech degree from the University of Lucknow (1992), his MEngg and $\mathrm{PhD}$ degrees from IIT Roorkee in 1997 and 2007, respectively. He is working in the Photogrammetry and Remote Sensing Department. His fields of expertise are soft computing, application for temporal images in specific class identification, digital photogrammetry, global positioning system, and LiDAR.

Kourosh Khoshelham is a lecturer at the Department of Infrastructure Engineering of the University of Melbourne. He received his MSc degree from the University of Tehran (2000) and his PhD at the Hong Kong Polytechnic University (2004). He worked at the University of Tehran, Delft University of Technology and ITC faculty of geoinformation science and Earth observation of the University of Twente, before moving to Melbourne in February 2015. His research interests include mobile mapping, indoor mapping, building information modeling, UAV photogrammetry, and information extraction from point clouds.

David B. James is a senior lecturer and head of the Sensors Group in the Centre for Electronic Warfare. He joined MoD in 1989 and worked on nonlinear optical systems and devices, gaining a PhD from the Royal Military College of Science in 2002. In 2008, he joined Cranfield University, conducting research into novel laser applications.

Mark A. Richardson is a professor of electronic warfare and is the director of research for Cranfield Defense and Security. He has his first-class BSc degree, his MSc degree with distinction, and his PhD. He is an associate of the Royal College of Science, has a Diploma of Imperial College, is an accredited senior imaging scientist and is a fellow of the Royal Photographic Society. His research interests lie within the use of electro-optics and infrared (EOIR) for electronic warfare, countermeasures, seekers, surveillance and target acquisition systems, man-portable air-defense systems (MANPADs), laser systems, digital image processing, electrooptical protective measures (EOPM), and electro-optical countermeasures (EOCM). 